\title{
The star formation rate in the Milky Way: Results from stars and planetary nebulae
}

\author{
Walter J. Maciel ${ }^{1}$, Helio J. Rocha-Pinto ${ }^{2}$, and Roberto D. D. Costa ${ }^{1}$ \\ ${ }^{1}$ Departmento de Astronomia, Universidade de São Paulo \\ Rua do Matão 1226, 05508-090 São Paulo SP, Brazil \\ email: maciel@astro.iag.usp.br, roberto@astro.iag.usp.br \\ ${ }^{2}$ Observatório do Valongo, Universidade Federal do Rio de Janeiro \\ Ladeira Pedro Antonio 43, 20080-090 Rio de Janeiro RJ, Brazil \\ email: helio@ov.ufrj.br
}

\begin{abstract}
The star formation rate (SFR) is still a poorly known characteristic of the Milky Way, especially concerning the possibility of an irregular SFR, compared to a constant rate. Some recent results based on the distribution of dwarf stars with chromospheric ages suggest at least two major bursts in the past $10 \mathrm{Gyr}$, while other investigations are consistent with an approximately constant SFR. The SFR also shows important spatial variations, particularly concerning the radial variations along the galactic disc. In this work, we investigate two different problems relative to the galactic SFR: (i) We estimate the star formation rate in the galactic disc based on the age distribution of the planetary nebula central stars (CSPN), and compare these results with previous investigations based on dwarf stars. The CSPN ages were derived on the basis of five different methods, involving the observed nebular metallicities and kinematical properties; (ii) We derive radial abundance gradients from several elements in planetary nebulae, and compare these results with recent determinations based on younger objects, such as HII regions and cepheid variables. Since the gradients are linked to the formation process of the galactic disc, we can estimate the spatial variation of the SFR. Preliminary results indicate that at least one major star formation burst is obtained, as well as a relatively smooth variation of the SFR along the galactocentric radius.
\end{abstract}

Keywords. Galaxy: formation, ISM: planetary nebuale

\section{Introduction}

Radial abundance gradients have long been considered as a key ingredient of the chemical evolution of spiral galaxies, especially when their magnitude, space and time variations are taken into account. These gradients are caused essentially by differences in the star formation rate in different regions of the galactic disc, so that they can give valuable information on the star formation process. We presently have detailed information on the gradients not only for the Milky Way, but also for several galaxies in the Local Group. Such information comes basically from young objects (HII regions, OB stars and associations, and cepheids), as well as from evolved objects (open clusters and planetary nebulae, PN). In this work we estimate the star formation rate (SFR) on the basis of (i) some recent determinations of the abundance gradients based on young as well as aged objects, and (ii) the determination of individual ages for a large sample of central stars of planetary nebulae (CSPN). 


\section{The CSPN age distribution}

We have made an effort to derive the age distrbution of the CSPN, in view of the fact that this information is essential in the interpretation of the abundance gradients and their time variations. Several methods have been developed based both on age-metallicity relations and on their kinematic properties (Maciel et al. 2010, 2011). Maciel et al. (2010) suggested that most objets studied have ages under $6 \mathrm{Gyr}$, and the average distribution peaks somewhere between 1 and $3 \mathrm{Gyr}$, depending on the assumptions made for the relation between the stellar masses and the corresponding ages. More recently, Maciel et al. (2011), based on kinematic ages of the CSPN, confirmed the previous results, but suggested generally lower ages for the CSPN.

\section{Average gradients in the galactic disc}

Gradients from young objects are observed in cepheids, HII regions, OB stars and associations. There are presently some controversies concerning the behviour of the gradients along the galactocentric radius, but an average gradient of -0.03 to $-0.06 \mathrm{dex} / \mathrm{kpc}$ is obtained for the Milky Way, with similar values for some of the galaxies in the Local Group. Concerning evolved objects, the most recent results apply to open clusters and planetary nebulae, and the gradients are typically in the range -0.03 to $-0.10 \mathrm{dex} / \mathrm{kpc}$ for oxygen and sulphur (Maciel et al. 2003, 2005, Henry et al. 2010, Andreuzzi et al. 2011).

A comparison of the oxygen abundances with Fe abundances, which are not well determined in $\mathrm{PN}$, indicates a tight correlation with a slope in the range 1.0 to 1.2 (Maciel 2002 ), so that $\mathrm{O} / \mathrm{H}$ and $\mathrm{S} / \mathrm{H}$ gradients from $\mathrm{PN}$ can be converted into $[\mathrm{Fe} / \mathrm{H}]$ gradients. The PN gradients are probably slightly larger than the gradients derived from the younger objects, but the difference seems now marginal compared to our previous investigations (Maciel et al. 2005), which may explain the essentially similar gradients measured in PN and HII regions in some Local Group galaxies (Stanghellini et al. 2010, Magrini et al. 2009) or in cepheids (Luck et al. 2011). The similarity of the gradients can be confirmed, for example, from the compilation by Spitoni \& Matteucci (2011). Taking into account the uncertainties in the determinations, it can be concluded that the abundance gradients have had a small change in the last 3-4 Gyr, slower than usually assumed. Earlier epochs are more difficult to assess, but results from older PN and open clusters (ages greater than 5 Gyr) generally suggest steeper gradients.

\section{Effects on the star formation rate}

(i) Recent investigations based on chromospheric ages of dwarf stars in the Milky Way disc suggest that the star formaton rate may have suffered time variations, especially in the last 5 or $6 \mathrm{Gyr}$, so that our Galaxy would have some characteristics resembling starburst galaxies. From the age distribution of the CSPN one can obtain some evidence of galactic bursts, assuming that the birth rate of the planetary nebulae is representative of the SFR in these systems. A first examination of the CSPN age distribution would suggest an enhanced rate a few Gyr ago, depending on the adopted hypotheses on the mass-age relationship. Figure 1a shows a comparison of the derived uncorrected SFR from Rocha-Pinto et al. (2000a,b) and the results from the present investigation. The dotted line refers to the original results by Rocha-Pinto et al. (2000b), while the solid and dashed lines show the SFR based on the PN age determinations from Maciel et al. (2011) and Maciel et al. (2010), respectively. It can be concluded that the age distribution of the CSPN supports the idea that at least one era of enhanced star formation activity 

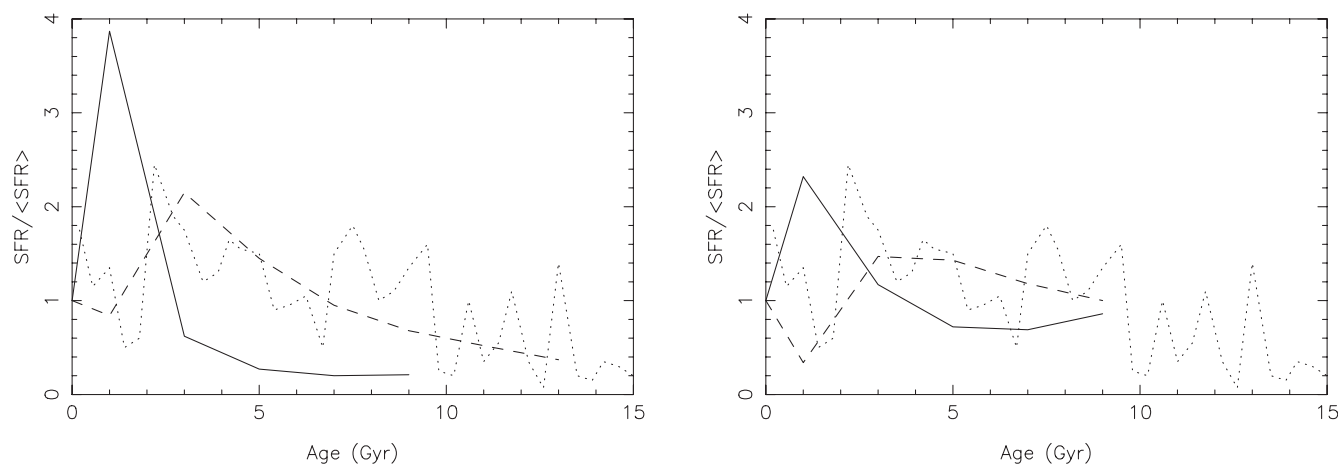

Figure 1. Time variation of the star formation rate. (a) uncorrected, (b) corrected. Dotted lines are the SFR by Rocha-Pinto et al. (2000), solid lines reflect results from Maciel et al. (2011), and the dashed lines are from the earlier work by Maciel et al. (2010).

occurred in the Milky Way in the last few Gyr. Figure 1b shows the SFR after applying a correction factor obtained using the initial mass function for the dying stars at each age interval. The peaks are somewhat decreased, but an important enhancement is still observed, especially for the kinematic ages.

(ii) Considering the similarity of the abundance gradients for young and evolved objects, it can be concluded that the average gradients have not changed much during the last 3 to $4 \mathrm{Gyr}$, approximately, depending on the adopted ages of the CSPN. Therefore, the SFR has probably not changed much as a function of the galactocentric radius during this period. Adopting an average gradient of $-0.05 \mathrm{dex} / \mathrm{kpc}$, and assuming that the abundance gradient is directly proportional to the SFR, the difference in the SFR along the galactic disc is about a factor of 4 in the region where the gradients are best measured, between the galactocentric distances $\mathrm{R}=4 \mathrm{kpc}$ and $\mathrm{R}=14 \mathrm{kpc}$, approximately. This implies a smooth variation of the SFR along the galactocentric radius, in very good agreement with the radial distribution of the SFR as summarized for example by Portinari \& Chiosi (1999).

\section{Acknowledgements}

This work was partially supported by FAPESP and CNPq.

\section{References}

Andreuzzi, G., Bragaglia, A., Tosi, M., \& Marconi, G. 2011, MNRAS 412, 1265

Henry, R. B. C., Kwitter, K. B., Jaskot, A. E., et al. 2010, ApJ 724, 748

Luck, R. E., Andrievsky, S. M., Kovtyukh, V. V., et al. 2011, AJ 142, 51

Maciel, W. J. 2002, Rev. Mex. A\&A SC 12, 207

Maciel, W. J., Costa, R. D. D., \& Idiart, T. E. P. 2010, A\&A 512, A19

Maciel, W. J., Costa, R. D. D., \& Uchida, M. M. M. 2003, A\& $A$ 397, 667

Maciel, W. J., Lago, L. J., \& Costa, R. D. D. 2005, A\&SA 433, 127

Maciel, W. J., Rodrigues, T. S., \& Costa, R. D. D. 2011, Rev. Mex. A\&SA, 47, 401

Magrini, L., Stanghellini, L., \& Villaver, E. 2009, ApJ 696, 729

Portinari, L. \& Chiosi, C. 1999, A\& A 350, 827

Rocha-Pinto, H. J., Scalo, J., Maciel, W. J., \& Flynn, C. 2000a, ApJ 531, L115

Rocha-Pinto, H. J., Scalo, J., Maciel, W. J., \& Flynn, C. 2000b, A\&SA 358, 869

Spitoni, E. \& Matteucci, F. 2011, A\& $A$ 531, A72

Stanghellini, L., Magrini, L., Villaver, E., \& Galli, D. 2010, A $\mathscr{U} A$ 521, A3 